\title{
Oxymatrine Liposomes for Intervertebral Disc Treatment: Formulation, in vitro and vivo Assessments
}

This article was published in the following Dove Press journal: Drug Design, Development and Therapy

\author{
Huan Wang ${ }^{l, *}$ \\ Yifan Ding ${ }^{1, *}$ \\ Wei Zhang ${ }^{2}$ \\ Kang Wei ${ }^{1}$ \\ Yaping Pei ${ }^{1}$ \\ Chenming Zou ${ }^{2}$ \\ Chong Zhang ${ }^{2}$ \\ Jiahui Ding ${ }^{2}$ \\ Huang Fang ' \\ Songwei Tan (iD) ${ }^{2}$ \\ 'Department of Orthopaedic Surgery, \\ Tongji Hospital, Tongji Medical College, \\ Huazhong University of Science and \\ Technology, Wuhan 430030, People's \\ Republic of China; ${ }^{2}$ Tongji School of \\ Pharmacy, Huazhong University of Science \\ and Technology, Wuhan 430030, People's \\ Republic of China \\ *These authors contributed equally to this \\ work
}

Purpose: Intervertebral disc degeneration (IVDD) is the main cause of modern low back pain, leading to high societal economic costs. To find an effective medical treatment for this disease, oxymatrine liposomes (OMT-LIP) were prepared with the pH-gradient method.

Materials and Methods: Nucleus pulposus (NP) cells from Sprague-Dawley rats were used for the cell experiments. Kunming mice were used for in vivo imaging. LIP were employed to deliver OMT, and the particle size, $\zeta$-potential, morphology, in vitro stability and in vitro release characteristics were evaluated. The OMT-LIP targeting effect was measured by in vivo imaging. Cell Counting Kit- 8 assays were used to detect the cytotoxicity of OMT and OMT-LIP on NP cells. Therapeutic efficacy was measured by Western blot, real-time quantitative polymerase chain reaction, and apoptosis assays. Radiologic analysis was performed to evaluate the therapeutic effects in vivo.

Results: Orthogonal test results revealed that the mass ratio of egg yolk phosphatidylcholine to cholesterol was the key factor to effectively trap OMT in LIP. Optimal OMT-LIP showed multivesicular structure with entrapment efficiency of $73.4 \pm 4.1 \%$, particle size of $178.1 \pm$ $2.9 \mathrm{~nm}$, and $\zeta$-potential of $-13.30 \pm 2.34 \mathrm{mV}$. OMT-LIP manifested excellent stability in vitro and presented significantly longer sustained release compared to OMT solution in phosphatebuffered saline ( $\mathrm{pH}$ 7.4). OMT-LIP conspicuously increased OMT accumulation in the degenerative disc, attenuated NP cell apoptosis, reduced the expression of matrix metalloproteinases 3/9 and interleukin-6, and decreased degradation of type II collagen. In in vivo study, X-ray demonstrated that OMT-LIP inhibited IVDD.

Conclusion: OMT-LIP may be a useful treatment to alleviate disc inflammation and IVDD. Keywords: intervertebral disc degeneration, liposomes, oxymatrine

\section{Introduction}

Low back pain (LBP) is one of the most common diseases leading to a low quality of life and high societal economic costs. ${ }^{1}$ The most direct cause of LBP is intervertebral disc (IVD) degeneration (IVDD). ${ }^{2}$ An imbalance of extracellular matrix (ECM) anabolism and catabolism in the nucleus pulposus (NP) induced by inflammation could lead to IVDD. ${ }^{3,4}$ During inflammation pathogenesis, matrix metalloproteinase (MMP) expression positively correlates with many inflammatory mediators such as interleukin (IL) $-1 \beta$ and the degradation of ECM components such as collagen type II (Col2). ${ }^{5}$ IL$1 \beta$ reportedly exacerbates the generation of apoptosis-associated proteins ${ }^{6}$ and various inflammatory molecules; ${ }^{7}$ this leads to apoptosis and fibrotic matrix synthesis and

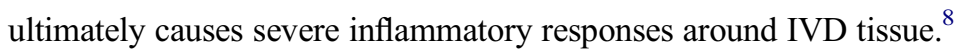

Correspondence: Huang Fang Department of Orthopaedic Surgery, Tongji Hospital, Tongji Medical College, Huazhong University of Science and Technology, Wuhan 430030, People's Republic of China

Email fanghuangtjh@hust.edu.cn

Songwei Tan

Tongji School of Pharmacy, Huazhong University of Science and Technology,

Wuhan 430030, People's Republic of

China

Email tansw@hust.edu.cn 
Oxymatrine (OMT) is derived from the roots of the traditional Chinese medicine named Sophora flavescensAit (Kushen). ${ }^{9}$ OMT exhibits antiviral, anti-fibrosis, and antiinflammatory effects and has been successfully used to treat chronic hepatitis B in clinical practice. ${ }^{10}$ Moreover, OMT can prevent synovial inflammation and migration in rheumatoid fibroblast-like synoviocytes. ${ }^{11}$ Since IVDD is also related to inflammation, OMT was thought to have great potential as an IVDD treatment. ${ }^{12}$

However, due to the wide biodistribution and rapid elimination after intravenous OMT injection, ${ }^{13}$ treatment is limited by low drug concentration and short residence time, especially since the IVD is the largest avascular tissue in the human body. ${ }^{14,15}$ Therefore, increasing OMT accumulation in IVD is crucial to improving therapeutic efficacy. Liposomes (LIP) are a biocompatible nanocarrier that have been employed to deliver various drugs. ${ }^{16}$ It improved the clinical applications of a wide range of drugs by stabilizing their structures, enhancing cellular internalization, and improving drug biodistribution in targeted areas. ${ }^{17,18}$ As a drug carrier system, LIP possess the following advantages: sustained release, ability for self-assembly, capacity to load large quantities of drugs, and other biophysical and physicochemical properties. ${ }^{16,18,19}$ We hypothesized that OMT LIP (OMT-LIP) could be used as an efficient intervention for IVDD.

In this study, OMT was loaded into LIP using a pHgradient method. Orthogonal testing was used to optimize the formula. The particle size, $\zeta$-potential, morphology, in vitro stability and in vitro release were evaluated. The in vitro stabilities of OMT-LIP stored at $4^{\circ} \mathrm{C}$ for 7 days or 3 months were investigated by particle size. Drug accumulation was detected by in vivo imaging. Hoechst 33258 staining and Annexin V-fluorescein isothiocyanate (FITC)/ propidium iodide (PI) doubling staining were performed to assess NP cell apoptosis. MMP3/9, IL-6, and Col2 mRNA and protein levels were measured by real-time quantitative polymerase chain reaction (RT-qPCR) and Western blot, respectively.

\section{Materials and Methods \\ Materials}

OMT (98\% purity) was purchased from Aladdin, Co., Ltd (Shanghai, China). Egg yolk phosphatidylcholine (EPC) was purchased from Sigma Aldrich (St. Louis, MO, USA). Cholesterol (Chol) was purchased from Avanti Polar Lipids, Inc. (Alabaster, AL, USA). mPEG2000-DSPE (DSPE) was purchased from Corden Pharma (Liestal, Switzerland). Highperformance liquid chromatography (HPLC)-grade acetonitrile was purchased from Fisher Chemical (Waltham, MA, USA). Cell Counting Kit-8 (CCK8) was purchased from Beyotime Biotechnology (Nanjing, China).

Kunming mice weighing 30-35 g and Sprague-Dawley rats weighing 200-250 g were both purchased from Beijing HFK Bioscience Co., Ltd (Beijing, China). All animal experimental procedures were performed in accordance with the International Guiding Principles for Animal Research and were approved by the Animal Experimentation Committee of Huazhong University of Science and Technology.

\section{OMT-LIP Preparation}

OMT-LIP were prepared by the pH-gradient method. Briefly, the lipids (EPC/Chol/DSPE) were dissolved in dichloromethane and dried to a thin film with a rotary evaporator under reduced pressure. Then, it was placed in a vacuum oven for $30 \mathrm{~min}$ for further drying. The lipid film was hydrated using an ultrasound machine with 0.1 $\mathrm{mol} / \mathrm{L}$ Lemon/Na buffer (pH 5.0). The obtained LIP were extruded three times through a polycarbonate membrane (pore size: $0.22 \mu \mathrm{m}$, Whatman, Maidstone, Kent, UK). After the formation of the empty LIP, the external $\mathrm{pH}$ was adjusted to $\mathrm{pH} 7.0$ with saturated $\mathrm{Na}_{2} \mathrm{HPO}_{4}$ solution. Then, OMT was added to the LIP solution. The LIP/OMT were then incubated for $1 \mathrm{~h}$ at $60^{\circ} \mathrm{C}$ with occasional shaking. After the solution cooled down, it was transferred into dialysis tubes (MWCO: 1000 Da) and dialyzed against phosphate-buffered saline (PBS, $\mathrm{pH}$ 7.4) to remove free OMT. The dialysate was changed every $30 \mathrm{~min}$ for a total of 3 times. After completion of dialysis, the resulting solution was centrifuged at $3000 \mathrm{r} / \mathrm{min}$ for $5 \mathrm{~min}$, and the bottom precipitate was discarded. The desired OMTLIP was obtained as the supernatant.

Factors affecting the encapsulation efficiency (EE) of OMT-LIP such as EPC/Chol and EPC/DSPE mass ratio were investigated with orthogonal testing.

\section{OMT-LIP Characterization}

Particle size and $\zeta$-potential of OMT-LIP were measured by dynamic light scattering (DLS; ZetaPlus, Brookhaven Instruments, Holtsville, NY, USA). OMT-LIP ultrastructure was determined using transmission electron micrography (TEM; JEM-1230, JEOL Ltd., Tokyo, Japan) after negative staining with sodium phosphotungstate solution $(2 \%, \mathrm{w} / \mathrm{v})$. The EE of OMT was detected with an HPLC system (mobile phase: mixture of $0.1 \mathrm{~mol} / \mathrm{L} \mathrm{KH}_{2} \mathrm{PO} 4$ solution [adjusted to 
pH 2.5 with phosphoric acid] and acetonitrile, 95:5; Hitachi, Tokyo, Japan) at a wavelength of $220 \mathrm{~nm}$. EE was calculated by the following formula:

$$
\begin{aligned}
\mathrm{EE}(\%) & =(\text { weight of OMT loaded }) \\
& /(\text { weight of OMT fed }) \times 100 \%
\end{aligned}
$$

In vitro OMT release was determined with the dialysis method. Briefly, $5 \mathrm{~mL}$ OMT-LIP was placed in the dialysis bag (MWCO: 1000) and immersed in $50 \mathrm{~mL}$ PBS (pH 7.4) at $37^{\circ} \mathrm{C}$ with constant shaking $(75 \mathrm{rpm})$. At pre-set time intervals, $1 \mathrm{~mL}$ of the dialysis solution was removed, and an equal volume of fresh media was added. OMT concentrations in the media were measured by HPLC. All experiments were repeated three times, and the results expressed as mean $\pm \mathrm{SD}(\mathrm{n}=3)$.

\section{Surgical Technique and Radiological Analysis}

The model operation was conducted in mice (IVDD group). After anesthetizing with $2 \%(\mathrm{w} / \mathrm{v})$ pentobarbital $(50 \mathrm{mg} / \mathrm{kg})$, the position of a tail disc at a specific level was determined by palpation on the caudal vertebra. Puncture was vertically performed on levels Co7-8 and Co8-9 of the coccyx with a $26 \mathrm{G}$ needle to a depth of $2 / 3$ of the disc thickness based on previous research. ${ }^{20}$ To eliminate the influence of different individuals on the experimental results, the rats punctured at disc Co7-8 were defined as the injection group $(n=15)$, those punctured at disc Co8-9 as the normal saline injection (IVDD) group ( $\mathrm{n}=15)$, and rats with non-punctured disc at Co910 as the control group $(\mathrm{n}=15)$. Moreover, the injection group was equally divided into OMT, LIP and OMT-LIP $(10 \mu \mathrm{L}, 100 \mu \mathrm{g} / \mu \mathrm{L}, \mathrm{n}=5)$ groups and the same amount of normal saline was injected at disc Co8-9. Radiographs were taken at a collimator-to-film distance of $66 \mathrm{~cm}$, an exposure of $63 \mathrm{mAs}$, and a penetration power of $65 \mathrm{kV}$. X-ray was performed on rats at 0,2 and 4 weeks after surgery. Disc heights were measured using the ImageJ software and expressed as the disc height index (DHI) using the method as previously described. ${ }^{21}$

\section{In vivo Imaging}

The in vivo distribution of OMT-LIP at the inflammation site was characterized using the fluorescent dye DiR with coupled LIP. Twelve female Kunming mice (30-35 g) were randomly divided into four groups (Control DiR, Puncture DiR, Control DiR-LIP, and Puncture DiR-LIP).
The Puncture group underwent the model operation. The first two groups were intraperitoneally injected with free $\operatorname{DiR}(300 \mu \mathrm{L}, 0.17 \mathrm{mg} / \mathrm{mL})$, and the latter two with DiRLIP $(300 \mu \mathrm{L}, 0.17 \mathrm{mg} / \mathrm{mL})$. Later, near-infrared fluorescent (NIRF) imaging of mice was performed with an in vivo imaging system (Pearl ${ }^{\circledR}$ Trilogy, LI-COR Inc., Lincoln, NE, USA) at different time points $(2,8,24$, and $36 \mathrm{~h}$ ) after the intervention.

\section{NP Cell Isolation and Culture}

NP cells were collected from the IVD tissue of SpragueDawley rats (200-250 g). First, the disc tissue was cut and separated with $0.25 \%$ trypsin (Biothink Biological Technology, Nanjing, China) including $0.01 \%$ EDTA for about $45 \mathrm{~min}$ at $37^{\circ} \mathrm{C}$. Then, we used $0.25 \% \mathrm{Col} 2$ (Gibco, Grand Island, NY, USA) to digest it for $3 \mathrm{~h}$ at $37^{\circ} \mathrm{C}$. Finally, cells were expanded in Dulbecco's minimum essential medium (DMEM)/F12 containing 10\% fetal bovine serum (FBS, Gibco) and 1\% penicillin/streptomycin (Biothink Biological Technology, Nanjing, China) at $37^{\circ} \mathrm{C}$ under $21 \% \mathrm{O}_{2}$ and $5 \%$ $\mathrm{CO}_{2}$. The NP cells used in the experiment were from the first three passages, and there were no obvious changes in cell morphology between the primary cells (passage 0 ) and later passaged cells (passage 2).

\section{Cell Viability Assay}

Cell viability was determined using CCK8 kits in accordance with the manufacturer's protocol. In brief, secondpassage NP cells were expanded in 96-well plates (1000 cells $/ \mathrm{cm}^{2}$ ) and incubated in DMEM/F12 with $10 \%$ FBS at $37^{\circ} \mathrm{C}$ for $24 \mathrm{~h}$. Then, OMT $(0,0.25,0.5$, and $1 \mathrm{mg} / \mathrm{mL})$ and OMT-LIP $(0,0.25,0.5$, and $1 \mathrm{mg} / \mathrm{mL})$ were added to the 96-well plates with cells and place in an incubator for $48 \mathrm{~h}$. After the intervention, the cells were washed twice with PBS, then each well was treated with $100 \mu \mathrm{L}$ of DMEM including $10 \mu \mathrm{L}$ of CCK-8 solution, and the plate was incubated for another $2 \mathrm{~h}$. The absorbance of the wells was then measured at $450 \mathrm{~nm}$ with a microplate reader.

\section{Apoptosis Analysis}

IL-1 $\beta$ was used to induce apoptosis in NP cells. They were seeded on sterile coverslips in 6-well plates. Upon reaching $90 \%$ confluence, the cells were treated for $72 \mathrm{~h}$ and analyzed with two methods. (1) Hoechst 33258 staining: Cells were washed twice with PBS and stained for $20 \mathrm{~min}$ at $37^{\circ} \mathrm{C}$ with Hoechst 33258 staining solution (Beyotime Biotechnology) in accordance with the manufacturer's 
protocol after the NP cell intervention. (2) Flow cytometry: NP cells were isolated and harvested using trypsin $(0.25 \%)$ and then washed twice with PBS. After treatment with the FITC Annexin V Apoptosis Detection Kit in line with the manufacturer's instructions (BD Pharmingen, Franklin Lakes, NJ, USA), NP cell apoptosis was analyzed by flow cytometry, and the ratios of apoptotic cells were calculated.

\section{RT-qPCR}

Total RNA was extracted from NP cells in 6-well plates using RNAiso Plus (Takara Bio Inc, Kusatsu, Japan). cDNA was synthesized using reverse transcriptase (Toyobo, Japan) with $2 \mu \mathrm{g}$ of total RNA in accordance with the manufacturer's instructions. The cDNA was amplified using specific primers and SYBR Premix Ex Tap (Tli RNaseH Plus) (2x) (Toyobo, Osaka, Japan). The cycle threshold $(\mathrm{Ct})$ values were collected and normalized to the level of the housekeeping gene glyceraldehyde 3-phosphate dehydrogenase (GAPDH). The relative mRNA levels of each target gene were measured with the $2^{-\Delta \Delta \mathrm{Ct}}$ method. The primer sequences are listed in Table 1.

\section{Western Blot}

Total protein extracted from NP cells was isolated by radioimmunoprecipitation assay buffer with $1 \%$ phenylmethylsulfonyl fluoride (Biothink Biological Technology), and then protein concentration was determined with the bicinchoninic acid protein assay kit (Biothink Biological Technology). The protein samples were separated by sodium dodecyl sulfate polyacrylamide gel electrophoresis and transferred to polyvinylidene fluoride membranes (Bio-Rad, Hercules, CA, USA). After blocking with 5\% nonfat milk, the membranes were

Table I Primer Sequences

\begin{tabular}{|l|l|l|}
\hline Gene Name & Forward $\left(\mathbf{5}^{\prime}\right.$-3') & Reverse $\mathbf{( 5 ^ { \prime } - \mathbf { 3 } ^ { \prime } )}$ \\
\hline MMP3 & $\begin{array}{l}\text { GCTCATCCTACCCAT } \\
\text { TGCAT }\end{array}$ & $\begin{array}{l}\text { GCTTCCCTGTCATC } \\
\text { TTCAGC }\end{array}$ \\
\hline MMP9 & $\begin{array}{l}\text { TCCTTGCAATGTGGAT } \\
\text { GTTT }\end{array}$ & $\begin{array}{l}\text { CGTCCTTGAAGAA } \\
\text { ATGCAGA }\end{array}$ \\
\hline IL-6 & $\begin{array}{l}\text { GAGGATACCACTCCCAAC } \\
\text { AGACC }\end{array}$ & $\begin{array}{l}\text { AAGTGCATCATC } \\
\text { GTTGTTCATACA }\end{array}$ \\
\hline Collagen 2 & $\begin{array}{l}\text { CTGGTGGAGCAGCAA } \\
\text { GAGC }\end{array}$ & $\begin{array}{l}\text { GTGGACAGTAGAC } \\
\text { GGAGGAAAG }\end{array}$ \\
\hline GAPDH & $\begin{array}{l}\text { GGTGAAGGTCGGTGTG } \\
\text { AACG }\end{array}$ & $\begin{array}{l}\text { CTCGCTCCTGGA } \\
\text { AGATGGTG }\end{array}$ \\
\hline
\end{tabular}

incubated with the diluted primary antibody on a shaker at $4^{\circ} \mathrm{C}$ overnight. Then, the membrane was washed with Trisbuffered saline with Tween three times for 10 min each and then incubated with the corresponding secondary antibody at $25^{\circ} \mathrm{C}$ for $1 \mathrm{~h}$. The proteins were visualized by enhanced chemiluminescence (Biothink Biological Technology) using the molecular imager Chemidoc xrs Imaging System (BioRad). The protein bands were analyzed with ImageJ software (version 1.48; National Institutes of Health, Bethesda, MD, USA). The primary antibodies used in this study are listed in Table 2 .

\section{Statistical Analysis}

Student's t-tests were used to compare two groups. Three or more groups were analyzed with one-way analysis of variance. For all tests, $p<0.05$ was considered statistically significant. Graphs were generated using GraphPrism software 7.0 (GraphPad Software Inc., San Diego, CA, USA). All data are expressed as mean \pm SD.

\section{Results \\ OMT-LIP Characterization}

LIP composed of EPC/Chol/DSPE obtained an EE of $73.4 \%$. The effects of lipid composition on EE were investigated, and the results are displayed in Tables 3 and 4. The range analysis showed that factor A (EPC:Chol) had the most significant effect on EE. The order of influencing factors was $\mathrm{A}>\mathrm{C}>\mathrm{B}$. The range of factor $\mathrm{B}$ was the smallest, with the least significant effect. Based on the influence of various factors on

Table 2 Primary Antibodies

\begin{tabular}{|l|l|l|l|l|}
\hline Name & Source & Brand & $\begin{array}{l}\text { Catalog } \\
\text { Number }\end{array}$ & Dilution \\
\hline GAPDH & Mouse & Proteintech & $60004-$ I-Ig & I:5000 \\
MMP3 & Mouse & Proteintech & $66338-$ I-Ig & I:5000 \\
MMP9 & Rabbit & Proteintech & 10375-2-AP & I:500 \\
IL-6 & Rabbit & Proteintech & 21865-I-AP & I:200 \\
Collagen 2 & Rabbit & Proteintech & I5943-I-AP & I:500 \\
\hline
\end{tabular}

Table 3 Levels of Various Factors

\begin{tabular}{|c|c|c|c|}
\hline \multirow[t]{2}{*}{ Level } & \multicolumn{3}{|c|}{ Factor } \\
\hline & A & B & C \\
\hline I & $3: 1$ & $3: 1$ & I \\
\hline 2 & $4: 1$ & 4:1 & 2 \\
\hline 3 & $5: 1$ & $5: 1$ & 3 \\
\hline
\end{tabular}

Abbreviations: A, EPC:Chol; B, EPC:DSPE; C, concentration of OMT (mg/mL). 
Table 4 Arrangement and Results of Orthogonal Test According to $\mathrm{L9}\left(3^{4}\right)$

\begin{tabular}{|l|l|l|l|l|}
\hline Number & A & B & C & EE\% \\
\hline 1 & $3: 1$ & $3: 1$ & 1 & 43.09 \\
2 & $3: 1$ & $4: 1$ & 2 & 33.99 \\
3 & $3: 1$ & $5: 1$ & 3 & 61.26 \\
4 & $4: 1$ & $3: 1$ & 2 & 63.34 \\
5 & $4: 1$ & $4: 1$ & 3 & 73.37 \\
6 & $4: 1$ & $5: 1$ & 1 & 58.91 \\
7 & $5: 1$ & $3: 1$ & 3 & 55.71 \\
8 & $5: 1$ & $4: 1$ & 1 & 43.82 \\
9 & $5: 1$ & $5: 1$ & 2 & 54.07 \\
KI & 138.34 & 162.14 & 145.82 & \\
K2 & 195.62 & 151.18 & 151.40 & \\
K3 & 153.60 & 174.24 & 190.34 & \\
R & 57.28 & 23.06 & 44.52 & \\
\hline
\end{tabular}

Abbreviations: A, EPC: Chol; B, EPC: DSPE; C, concentration of OMT (mg/mL) $E E$, encapsulation efficiency.

$\mathrm{EE}$, the optimized preparation conditions were determined as $\mathrm{A}_{2} \mathrm{~B}_{2} \mathrm{C}_{3}$, that is, the concentration of OMT was $3 \mathrm{mg} / \mathrm{mL}$, and the mass ratio of EPC:Chol:DSPE was 4:1:1.

The morphology of OMT-LIP was found to be multivesicular structure with a spherical shape (Figure 1A). The OMTLIP particle size was found to be $178.1 \pm 2.9 \mathrm{~nm}$ with a polydispersity index of $0.167 \pm 0.034$ and $\zeta$-potential of $13.30 \pm 2.34 \mathrm{mV}$ (Figure 1B). After incubation for 7 days or 3 months at $4^{\circ} \mathrm{C}$, the formulated LIP showed minimal size change (Figure 1C and D). These results suggest that OMTLIP exhibit excellent physical stability.

Next, the in vitro drug release for a 48 -h period was evaluated; samples were collected at 1, 3, 5, 7, 9, 11, 24, 36 , and $48 \mathrm{~h}$ (Figure 1E). The cumulative release of OMT was $67.2 \pm 3.0 \%$ in $48 \mathrm{~h}$, suggesting that OMT-LIP could achieve sustained release in vitro and increase the acting time of the drug compared to the immediate and spontaneous release of free OMT. The drug-release mechanism was further investigated. Table 5 shows that the in vitro drug-release kinetic model for OMT-LIP in PBS ( $\mathrm{pH} 7.0)$ fits well with Weibull's equation.

\section{Degenerative IVD Targeting}

To investigate the targeting effect of LIP in IVDD model mice after intraperitoneal administration, DiR dye was incorporated during liposome preparation. Figure 2 shows the images of the control and puncture groups after treatment with DiR or DiRLIP at different time points $(2,8,24$, and $36 \mathrm{~h})$. The images demonstrated that fluorescence intensity in the Puncture DiRLIP group gradually ( $2 \mathrm{~h}$ ) accumulated at the inflamed IVD, with a more obvious fluorescence signal later on (36 h) (Figure 2A). However, the other three groups did not show specific fluorescence intensity enhancement compared to the Puncture DiR-LIP group (Figure 2B and C).
A

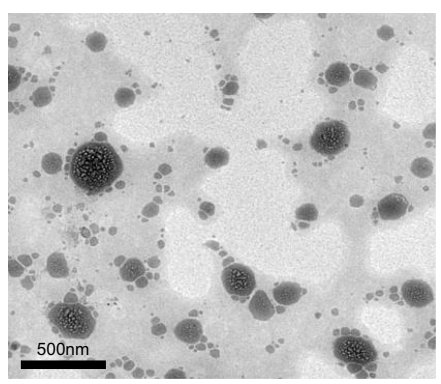

D

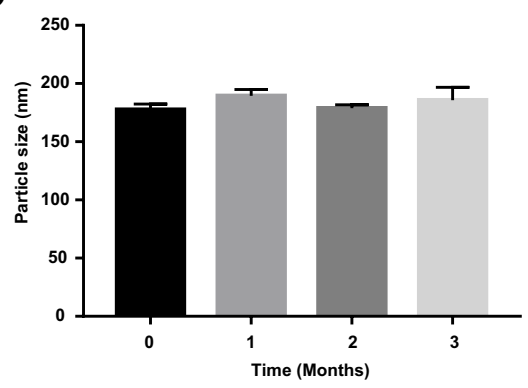

B

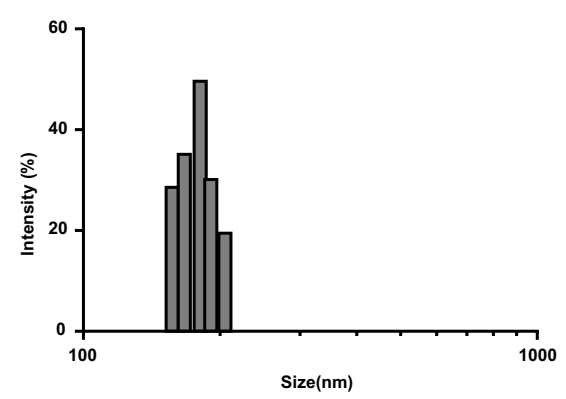

E

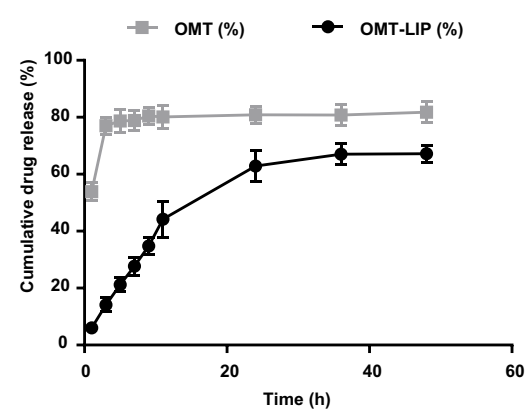

C

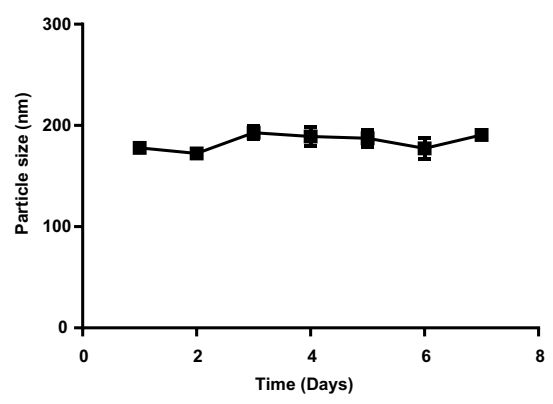

Figure I OMT-LIP characteristics. (A) TEM image of OMT-LIP by negative staining with sodium phosphotungstate solution (50,000x). (B) Particle size of OMT-LIP. (C-D) Particle size of OMT-LIP during storage at $4^{\circ} \mathrm{C}$ for 7 days and 3 months. (E) In vitro release of OMT-LIP and free OMT in PBS (pH 7.4). Scale bar $=500 \mathrm{~nm}$. 
Table 5 Release Kinetic Parameters for OMT from OMT-LIP in PBS (pH 7.4)

\begin{tabular}{|l|l|}
\hline & OMT-Loaded Liposome \\
\cline { 2 - 2 } & Equation Correlation Coefficient (R) \\
\hline Zero-order equation & $\mathrm{Q}=0.0 \mathrm{I} 2 \mathrm{It}+0.13350 .8786$ \\
$\begin{array}{l}\text { First-order equation } \\
\text { Higuchi }\end{array}$ & $\mathrm{Ln}(\mathrm{I}-\mathrm{Q})=-0.0204 \mathrm{t}-0.12030 .9346$ \\
Weibull's equation & $\mathrm{Q}=0.1047 \mathrm{t}_{\mathrm{I} / 2}-0.04 \mathrm{I} 0.9677$ \\
$\operatorname{Ln} \ln (\mathrm{I} /[\mathrm{I}-\mathrm{Q}])=0.805 \mathrm{Int}-2.9440 .9863$ \\
\hline
\end{tabular}

\section{OMT-LIP Protected NP Cells Against IL- I $\beta$-Induced Apoptosis}

The cytotoxic effects of OMT and OMT-LIP on NP cells were first determined at various concentrations $(0,0.25$, 0.5 , and $1 \mathrm{mg} / \mathrm{mL}$ ) for $48 \mathrm{~h}$. As shown in Figure $3 \mathrm{~A}$, proliferation decreased very slightly with increasing OMT concentration up to $1 \mathrm{mg} / \mathrm{mL}$. Therefore, $1 \mathrm{mg} / \mathrm{mL}$ OMT-LIP was used for subsequent experiments. To evaluate the effect of OMT-LIP on IL- $1 \beta$-induced apoptosis, Hoechst 33258 staining and flow cytometry were used to observe the morphology and apoptotic rate of NP cells, respectively. As shown in Figure 3B, obvious morphological changes and up-regulation of nuclear fragmentation occurred after stimulation with IL-1 $\beta$ (10 ng/mL). OMTLIP (1 mg/mL) significantly attenuated this phenomenon. Next, apoptosis markers were detected by flow cytometry (Figure $3 \mathrm{C}$ and $\mathrm{D}$ ). The results showed that the rate of apoptosis significantly increased under IL-1 $\beta$ induction compared with the normal group; however, this was significantly attenuated by OMT-LIP. These data indicated that OMT-LIP prevented IL-1 $\beta$-induced apoptosis of NP cells.

\section{OMT-LIP Inhibited IL-I $\beta$-Induced Increases in MMP and IL-6 Expression and Col2 Loss in NP Cells}

To evaluate the effects of OMT-LIP on ECM components in NP cells, the protein levels of matrix-degrading enzymes (MMP3 and MMP9) and a matrix component (Col2) were detected. As shown in Figure 4A and B, IL$1 \beta$ increased the expression of MMP3/9 and IL- 6 but decreased that of Col2. However, OMT-LIP $(1 \mathrm{mg} / \mathrm{mL})$ reduced the loss of $\mathrm{Col} 2$ and inhibited the expression of MMP3/9 and IL-6. The transcript levels of MMP3/9, IL-6, and Col2 in NP cells were detected by RT-qPCR after IL$1 \beta(10 \mathrm{ng} / \mathrm{mL})$ treatment and OMT-LIP intervention. As shown in Figure 4C, mRNA levels of MMP3/9 and IL-6 were obviously upregulated and Col2 was decreased after the addition of IL-1 $\beta$. Following OMT-LIP treatment, we observed obvious downregulation of MMP3/9 and IL-6 and recovery of $\mathrm{Col} 2$ (Figure 4C). These data indicated that OMT-LIP suppressed the expression of catabolic enzymes and promoted ECM synthesis.

\section{OMT-LIP Prevented IVDD in vivo}

To examine whether OMT-LIP has protective effects on degenerative intervertebral disc, we had the caudal vertebrae of rats underwent X-ray inspection. Prior to the puncture ( 0 weeks), all groups showed normal disc height (Figure 5A). However, the X-ray results at 2 weeks revealed a loss of disc height in IVDD group, indicating progressive degeneration. In contrast, OMT-LIP injection delayed its loss conspicuously (Figure 5A). At 4 weeks, the same results could be found that OMT-LIP group slowed down the narrowing of disc height and the percent disc height index (\%DHI) in the OMT and OMT-LIP treatment groups was 1.39- and 1.75fold higher than that in the IVDD group (Figure 5B). These results suggested that OMT-LIP treatment could alleviate the progression of IVDD in vivo.

\section{Discussion}

Currently, the main treatments for IVDD are pharmacologic interventions and surgery. ${ }^{22}$ The challenges for effective treatment are low drug concentration and inability to accumulate at the IVD tissue. Here, we developed OMTLIP to increase local drug concentration and enhance the efficacy of interventions for IVDD. Increasing the EE of water-soluble drug LIP has always been one of the difficulties in their preparation. ${ }^{23}$ OMT is a small molecule with high water solubility. The EE of liposome was $<20 \%$ by ethanol injection but reached $>50 \%$ with the $\mathrm{pH}$ gradient method. ${ }^{24}$ In our study, LIP composed of EPC/ Chol/DSPE achieved a maximum EE of $73.4 \%$. Citrate buffer was used during drug preparation. Citrate is a $\mathrm{pH}$ gradient used in the drug-loading process for weakly basic drugs, ${ }^{25}$ and this markedly improved OMT entrapment. ${ }^{26}$

Higher EE occurs in LIP with a moderate mass ratio of EPC to Chol (4:1) compared to the groups with mass ratios of $3: 1$ and 5:1 (Table 4). Chol influences the mechanical properties of cell membranes, increasing its mechanical strength, elasticity, and density through ordering and condensing effects. ${ }^{27-29}$ Due to the above modifications, small molecules and ions have less access to the membrane with increasing Chol. ${ }^{30}$ Therefore, a suitable Chol concentration is needed to achieve maximum EE of the drug. 
A

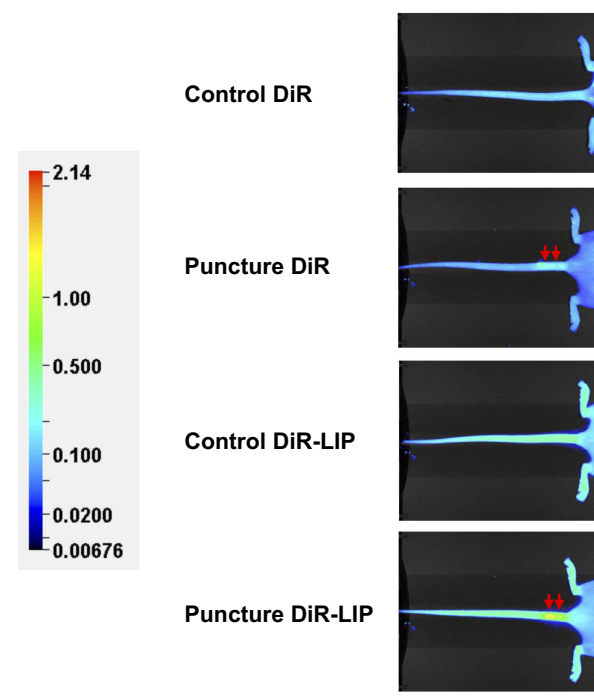

2h

B
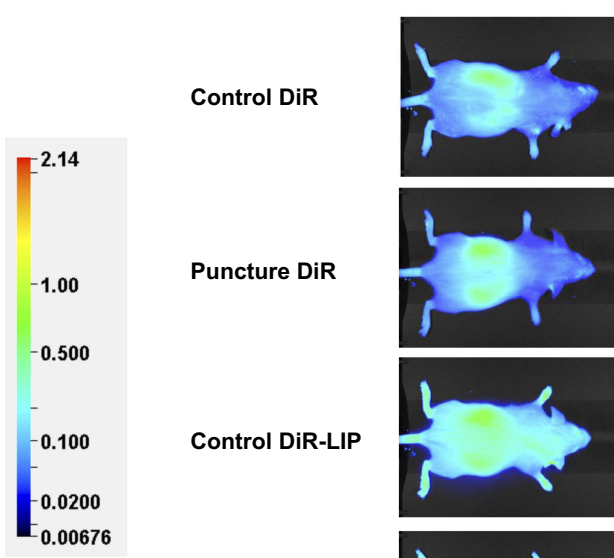

Puncture DiR
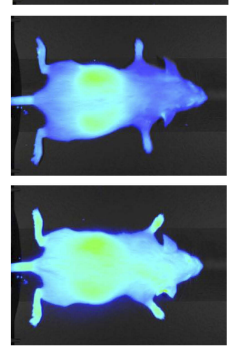

Control DiR-LIP

Puncture DiR-LIP

Puncture DiR-LIP

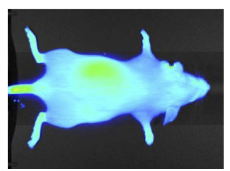

$2 h$

C

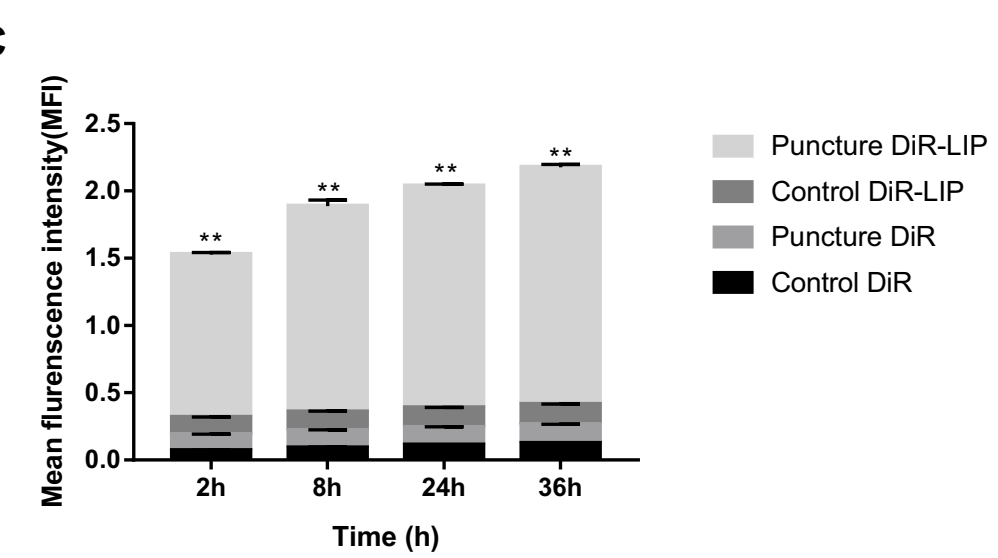

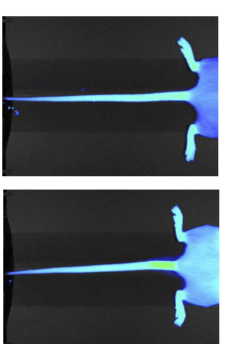
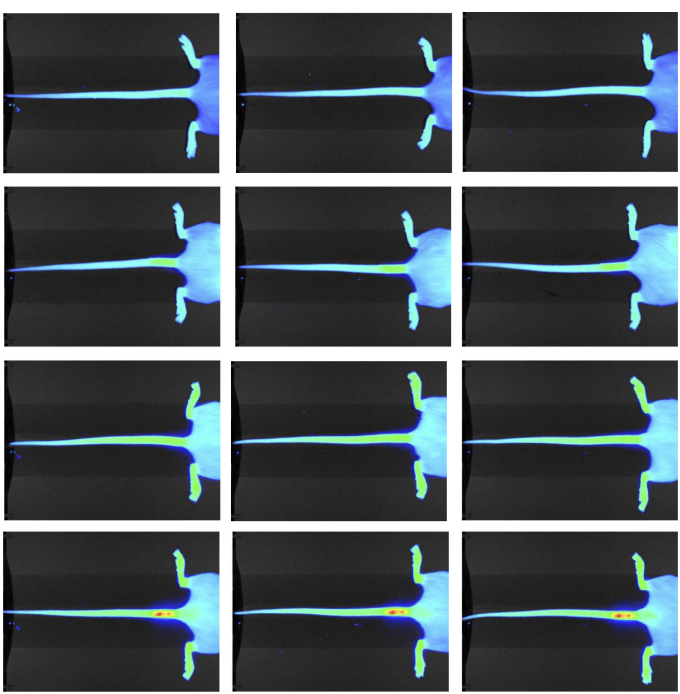

$8 h$

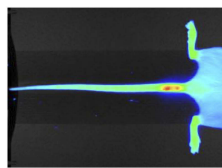

24h

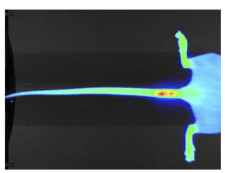

$36 \mathrm{~h}$
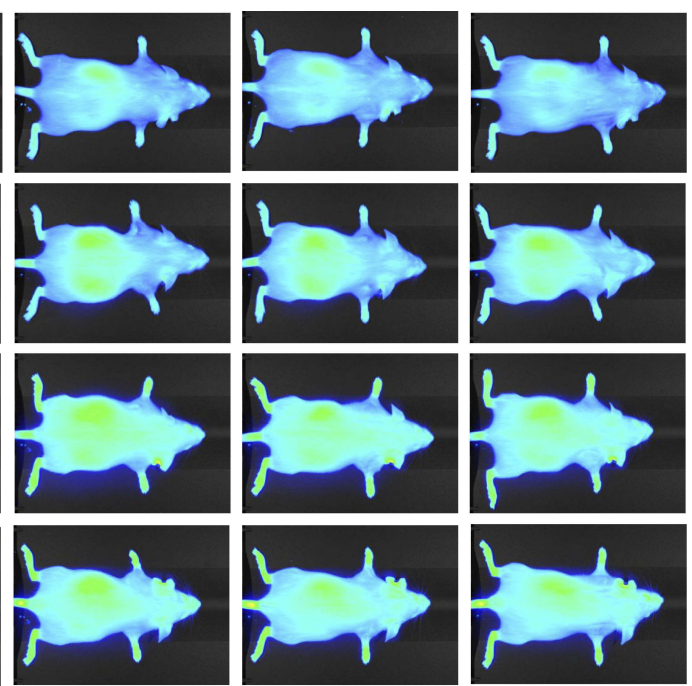

$8 h$

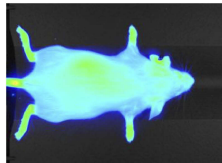

24h

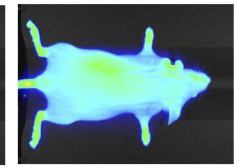

$36 \mathrm{~h}$

Figure 2 Biodistribution of the liposome delivery system was detected by NIRF imaging. The images were obtained after intraperitoneal administration of free DiR or DiR fabricated with LIP at different time intervals (2, 8, 24, and 36 h). (A) Tail images. (B) Whole-body images. (C) Histogram of quantitative analysis of mean fluorescence intensity at the degenerative intervertebral discs. Red arrows indicate puncture sites. ${ }^{*} p<0.01$ vs Puncture DiR group, $n=3$. 

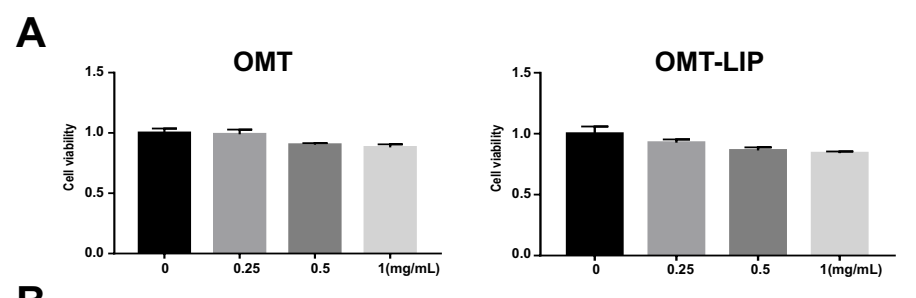

B
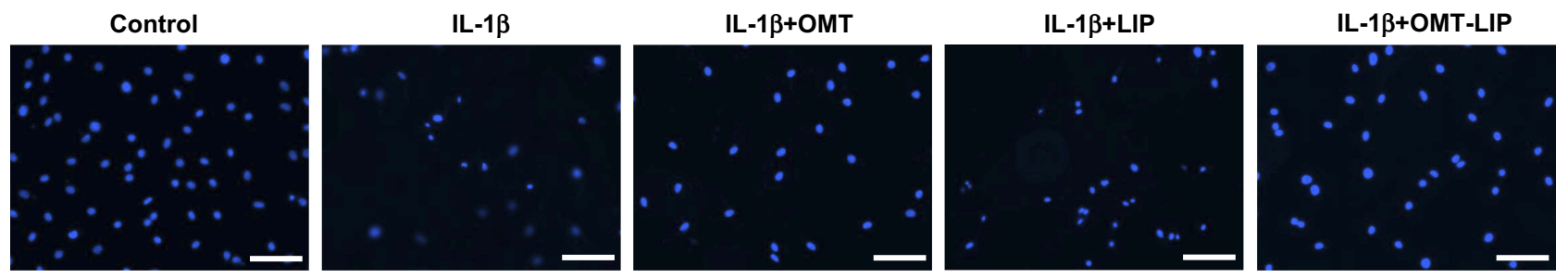

C

Control

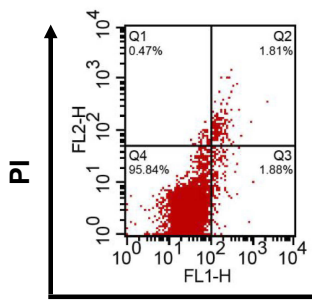

IL-1 $\beta$

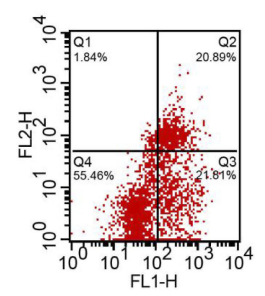

IL-1 $\beta+O M T$

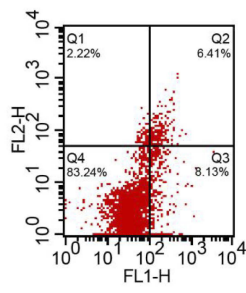

IL-1 $\beta+$ LIP

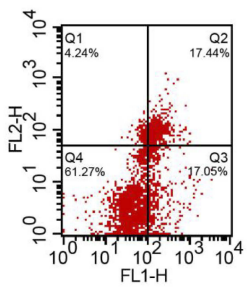

IL-1 $\beta+O M T-L I P$

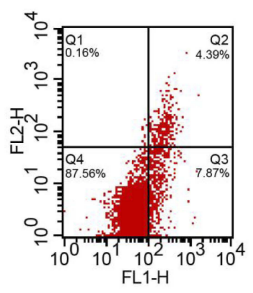

FITC-AnnexinV

D
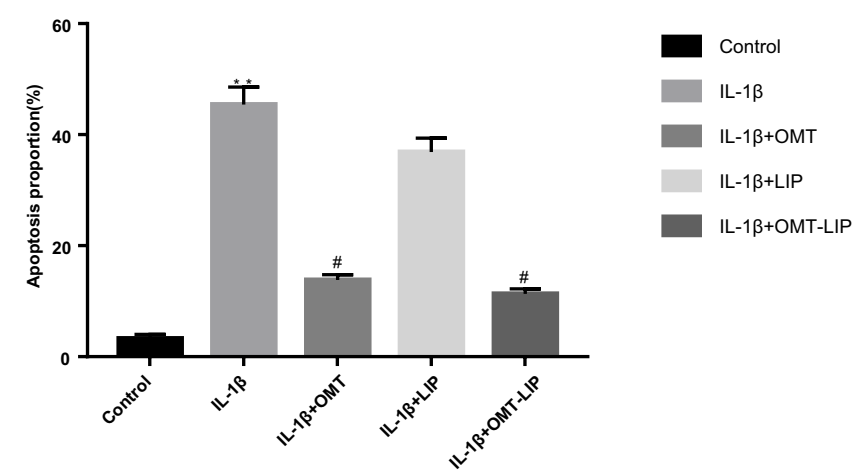

Figure 3 OMT-LIP ameliorated IL-I $\beta$-induced apoptosis in NP cells. (A) CCK-8 results of NP cells treated with different concentrations of OMT and OMT-LIP for 48 h. (B) Representative images for nuclei staining with Hoechst 33258 of NP cells observed by fluorescence microscope. (C) NP cell apoptosis was evaluated by flow cytometric analysis after Annexin-V/PI double staining. (D) Quantitative analysis of apoptosis by flow cytometry. Scale bar $=200 \mu \mathrm{m}$. ** $p<0.0 \mathrm{I}$ vs Control group, $\mathrm{n}=5$; \#p $<0.05 \mathrm{vs} \mathrm{IL-I \beta}$ group, $\mathrm{n}=5$.

In vivo imaging demonstrated that LIP could target degenerative IVDs. LIP were previously shown to exhibit targeting capability in a rat model of osteoarthritis. ${ }^{31}$ When LIP enter circulation, they are easily phagocytosed by macrophages as foreign bodies, meaning they naturally target the monocyte-macrophage-enriched organs of the liver and spleen. ${ }^{32}$ Macrophages accumulate at sites of inflammation where they participate in the inflammatory response. $^{33}$ This may explain why LIP have a role in inflammation targeting. Among the four groups, puncture
DiR-LIP mice showed a gradual and specific accumulation of fluorescence signal where the surgery was performed. This was not observed in the other three groups, excluding an effect of DiR or injection method. In summary, LIP effectively targeted IVD.

Nano-LIP are the most commonly used nano-carrier to deliver drugs to human tissues in clinical applications and have been approved by the US Food and Drug Administration. ${ }^{34}$ OMT has been reported to ameliorate agomelatine-induced hepatocyte toxicity ${ }^{35}$ and is safe for the 
A

IL-6

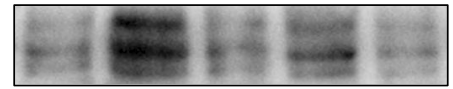

MMP3
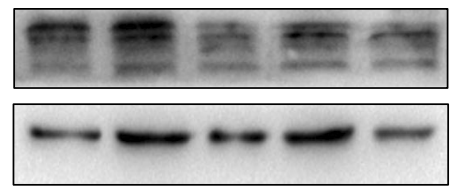

Col2

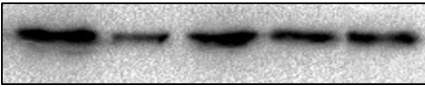

GAPDH

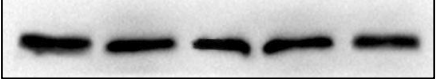

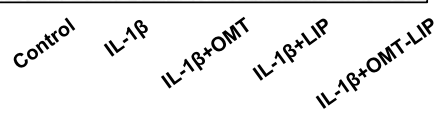

B

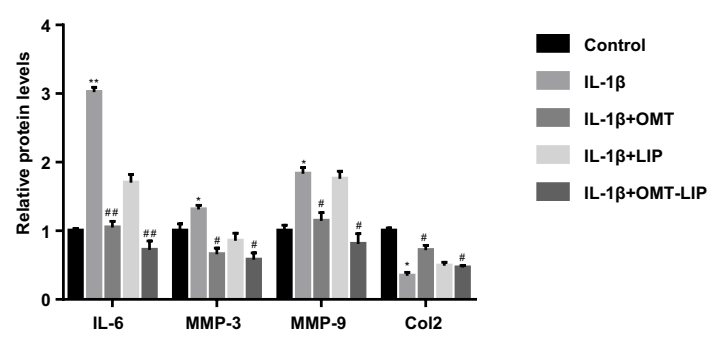

C

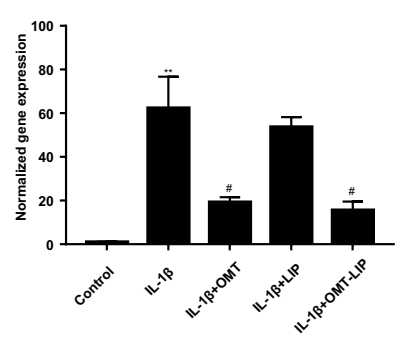

MMP3

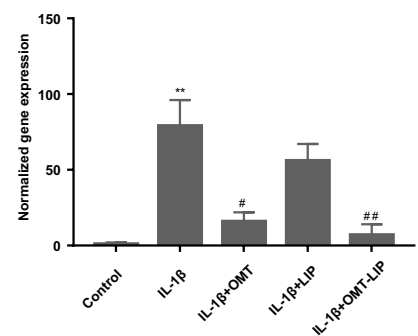

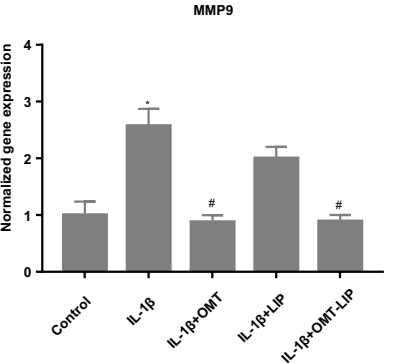

Col2

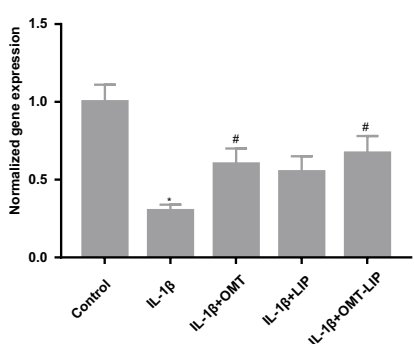

Figure 4 OMT-LIP inhibited MMP3/9, IL-6, and Col2 expression at mRNA and protein levels. (A) Western blot results for MMP3/9, IL-6, and Col2. (B) Band densitometric analysis. (C) mRNA levels of MMP3/9, IL-6, and Col2 as determined by RT-qPCR. The values are presented as mean \pm SD. $* p<0.05$, **p $<0.01$ vs Control group, $n=6$; \#p $<0.05$, $\#$ \# $<0.01$ vs IL-I $\beta$ group, $\mathrm{n}=6$.

A

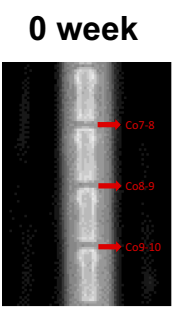

LIP
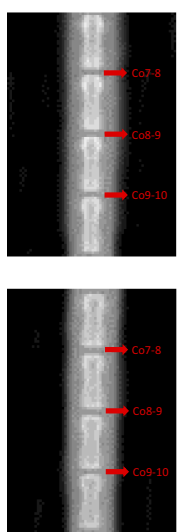

OMT-LIP
$\mathbf{B}$
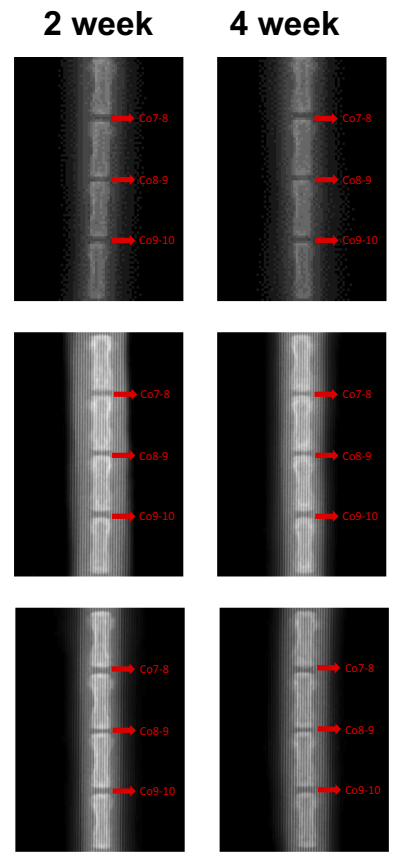
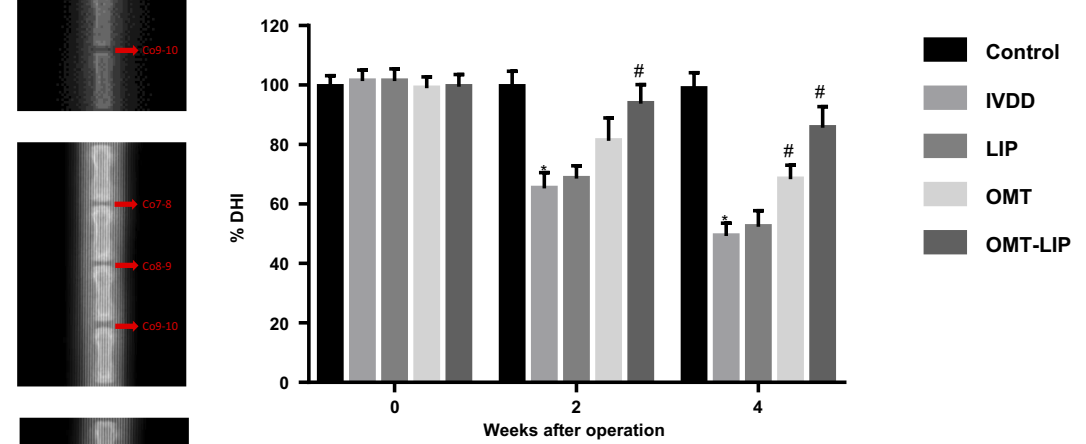

Figure 5 X-ray radiological analysis manifested OMT-LIP could alleviate IVDD in vivo. (A) X-ray results of 0,2 and 4 weeks after disc $p$,
changes of $\mathrm{DHI}$ at 0,2 and 4 weeks after disc puncture surgery. ${ }^{*} p<0.05$ vs Control group, $n=5 ; \# p<0.05$ vs IVDD group, $n=5$.

(B) The sequential 作 
treatment of hepatitis B cirrhosis. $^{36}$ Furthermore, OMT improved lesions in patients with severe psoriasis without obvious toxicity. ${ }^{37} \mathrm{CCK} 8$ assays confirmed no obvious toxicity of OMT-LIP to NP cells, indicating that both OMT and OMT-LIP are safe within a reasonable concentration gradient (Figure 3A). Furthermore, OMT-LIP obviously repressed IL$1 \beta$-induced apoptosis detected by fluorescence microscope and flow cytometry, as shown in Figure 3B-D.

Inflammation plays an important role in IVDD pathogenesis. In this context, MMPs and IL-6 could be upregulated by pro-inflammatory cytokines such as IL-1 $\beta$, leading to the decreased expression of ECM components such as Col2. We found that OMT-LIP markedly reduced the mRNA and protein levels of MMP3/9 and IL-6 and mitigated ECM degeneration, indicating that OMT-LIP obviously inhibited IL-1 $\beta$-induced disc degeneration (Figure 4).

Although OMT has clear therapeutic effects in many diseases, its mechanisms are still not completely understood. NF- $\kappa \mathrm{B}$ is a nuclear transcription factor that enters the nucleus upon activation to regulate the expression of inflammation-associated genes in response to injury or stress. OMT may enhance the expression of $\mathrm{I} \kappa \mathrm{B} \alpha$ to prevent NF- $\kappa \mathrm{B}$ from translocating into the nucleus, thus inhibiting NF- $\mathrm{B}$ signaling. ${ }^{38,39}$ One group reported that OMT ameliorated 1-arginine-induced acute pancreatitis and intestine injury through down-regulating $\mathrm{MAPK} / \mathrm{NF}-\kappa \mathrm{B}$ activation. $^{40}$ In addition, OMT could prevent synovial inflammation by blocking the NF- $\mathrm{B}$ pathway. ${ }^{11}$ Hence, OMT may attenuate IVDD by suppressing NF- $\kappa$ B signaling. Further studies are needed to fully understand the beneficial effects of OMT. In in vivo study, we demonstrated that OMT-LIP could effectively ameliorate the exacerbation of IVDD by X-ray radiological analysis. Overall, the OMT-LIP formulation showed desirable physical stability and properties, with high loading efficiency, appropriate particle size, and $\zeta$-potential for degenerative intervertebral disc targeting. And also, OMT-LIP could increase drug accumulation in the intervertebral disc. The study demonstrated that OMT-LIP ameliorated NP cell apoptosis and the ECM degeneration, providing a new perspective for IVDD treatment.

\section{Acknowledgments}

This work is supported by Natural Science Foundation of China (No.81271347 and 81871473). The authors thank the Analytical and Testing Centre of HUST for TEM measurements.

\section{Author Contributions}

HF and SWT conceived and designed research. HW and YFD conducted experiments. WZ, KW, YPP and CMZ contributed analytical tools. $\mathrm{CZ}$ and JHD analyzed data. HF wrote the manuscript. All authors contributed to data analysis, drafting or revising the article, gave final approval of the version to be published, and agree to be accountable for all aspects of the work.

\section{Disclosure}

The authors report no conflicts of interest in this work.

\section{References}

1. Lin Y, Jiao Y, Yuan Y, et al. Propionibacterium acnes induces intervertebral disc degeneration by promoting nucleus pulposus cell apoptosis via the TLR2/JNK/mitochondrial-mediated pathway. Emerg Microbes Infect. 2018;7(1):1.

2. Bez M, Zhou Z, Sheyn D, et al. Molecular pain markers correlate with $\mathrm{pH}$-sensitive MRI signal in a pig model of disc degeneration. Sci Rep. 2018;8(1):17363. doi:10.1038/s41598-018-34582-6

3. Wang H, He P, Pan H, et al. Circular RNA circ-4099 is induced by TNF- $\alpha$ and regulates ECM synthesis by blocking miR-616-5p inhibition of Sox9 in intervertebral disc degeneration. Exp Mol Med. 2018;50(4):27. doi:10.1038/s12276-018-0056-7

4. Kwon WK, Moon HJ, Kwon TH, Park YK, Kim JH. The role of hypoxia in angiogenesis and extracellular matrix regulation of intervertebral disc cells during inflammatory reactions. Neurosurgery. 2017;81(5):867-875. doi:10.1093/neuros/nyx149

5. Jabłońska-Trypuć A, Matejczyk M, Rosochacki S. Matrix metalloproteinases (MMPs), the main extracellular matrix (ECM) enzymes in collagen degradation, as a target for anticancer drugs. $J$ Enzyme Inhib Med Chem. 2016;31(sup1):177-183. doi:10.3109/147563 66.2016.1161620

6. Liu Q, Zhao G, Zhou C, et al. Oxaloacetate and adipose stromal cells-conditional medium synergistically protected potassium/serum deprivation-induced neuronal apoptosis. Brain Res Bull. 2017;128:7-12. doi:10.1016/j.brainresbull.2016.11.002

7. Molina-Holgado E, Ortiz S, Molina-Holgado F, Guaza C. Induction of COX-2 and PGE(2) biosynthesis by IL-1beta is mediated by PKC and mitogen-activated protein kinases in murine astrocytes. $\mathrm{Br}$ $J$ Pharmacol. 2000;131(1):152-159. doi:10.1038/sj.bjp.0703557

8. Tu J, Li W, Zhang Y, et al. Simvastatin inhibits IL-1ß-Induced apoptosis and extracellular matrix degradation by suppressing the NF-kB and MAPK pathways in nucleus pulposus cells. Inflammation. 2017;40(3):725-734. doi:10.1007/s10753-017-0516-6

9. Sang X, Wang R, Han Y, et al. T cell-associated immunoregulation and antiviral effect of oxymatrine in hydrodynamic injection HBV mouse model. Acta Pharm Sin B. 2017;7(3):311-318. doi:10.1016/j. apsb.2017.02.005

10. Li Y, Wang G, Liu J, Ouyang L. Quinolizidine alkaloids derivatives from Sophora alopecuroides Linn: bioactivities, structure-activity relationships and preliminary molecular mechanisms. Eur J Med Chem. 2019;188:111972. doi:10.1016/j.ejmech.2019.111972

11. Liang J, Chang B, Huang M, et al. Oxymatrine prevents synovial inflammation and migration via blocking NF- $\mathrm{kB}$ activation in rheumatoid fibroblast-like synoviocytes. Int Immunopharmacol. 2018;55:105-111. doi:10.1016/j.intimp.2017.12.006

12. Dai JP, Wang QW, Su Y, et al. Oxymatrine inhibits influenza A virus replication and inflammation via TLR4, p38 MAPK and NF- $\mathrm{KB}$ pathways. Int J Mol Sci. 2018;19(4):965. doi:10.3390/ijms19040965 
13. Wu XL, Hang TJ, Shen JP, Zhang YD. Determination and pharmacokinetic study of oxymatrine and its metabolite matrine in human plasma by liquid chromatography tandem mass spectrometry. J Pharm Biomed Anal. 2006;41(3):918-924. doi:10.1016/j.jpba.2006.01.029

14. Amelot A, Mazel C. The intervertebral disc: physiology and pathology of a Brittle joint. World Neurosurg. 2018;120:265-273. doi:10.1016/j.wneu.2018.09.032

15. Molinos M, Almeida CR, Caldeira J, Cunha C, Gonçalves RM, Barbosa MA. Inflammation in intervertebral disc degeneration and regeneration. $J \quad R$ Soc Interface. 2015;12(104):20141191. doi:10.1098/rsif.2014.1191

16. Sercombe L, Veerati T, Moheimani F, Wu SY, Sood AK, Hua S. Advances and challenges of liposome assisted drug delivery. Front Pharmacol. 2015;6:286. doi:10.3389/fphar.2015.00286

17. Metselaar JM, Storm G. Liposomes in the treatment of inflammatory disorders. Expert Opin Drug Deliv. 2005;2(3):465-476. doi:10.1517/ 17425247.2.3.465

18. Hua S, Wu SY. The use of lipid-based nanocarriers for targeted pain therapies. Front Pharmacol. 2013;4:143. doi:10.3389/ fphar.2013.00143

19. Pachis K, Blazaki S, Tzatzarakis M, et al. Sustained release of intravitreal flurbiprofen from a novel drug-in-liposome-in-hydrogel formulation. Eur J Pharm Sci. 2017;109:324-333. doi:10.1016/j. ejps.2017.08.028

20. Tian Z, Ma X, Yasen M, et al. Intervertebral disc degeneration in a percutaneous mouse tail injury model. Am J Phys Med Rehabil. 2018;97(3):170-177. doi:10.1097/PHM.0000000000000818

21. Wu X, Liu Y, Guo X, et al. Prolactin inhibits the progression of intervertebral disc degeneration through inactivation of the $\mathrm{NF}-\kappa \mathrm{B}$ pathway in rats. Cell Death Dis. 2018;9(2):98. doi:10.1038/s41419. 017-0151-z

22. Henry N, Clouet J, Le Bideau J, Le Visage C, Guicheux J. Innovative strategies for intervertebral disc regenerative medicine: from cell therapies to multiscale delivery systems. Biotechnol Adv. 2018;36 (1):281-294. doi:10.1016/j.biotechadv.2017.11.009

23. Li Q, Cai T, Huang Y, Xia X, Cole SPC, Cai Y. A review of the structure, preparation, and application of NLCs, PNPs, and PLNs. Nanomaterials (Basel, Switzerland). 2017;7(6). doi:10.3390/ nano7120458

24. Du S, Deng Y. Studies on the encapsulation of oxymatrine into liposomes by ethanol injection and $\mathrm{pH}$ gradient method. Drug Dev Ind Pharm. 2006;32(7):791-797. doi:10.1080/03639040600760556

25. Zucker D, Marcus D, Barenholz Y, Goldblum A. Liposome drugs' loading efficiency: a working model based on loading conditions and drug's physicochemical properties. J Control Release. 2009;139 (1):73-80. doi:10.1016/j.jconrel.2009.05.036

26. Zhang $\mathrm{S}$, Wu J, Wang $\mathrm{H}$, et al. Liposomal oxymatrine in hepatic fibrosis treatment: formulation, in vitro and in vivo assessment. AAPS PharmSciTech. 2014;15(3):620-629. doi:10.1208/s12249-014-0086-y

27. Magarkar A, Dhawan V, Kallinteri P, et al. Cholesterol level affects surface charge of lipid membranes in saline solution. Sci Rep. 2014;4:5005. doi:10.1038/srep05005
28. Briuglia ML, Rotella C, McFarlane A, Lamprou DA. Influence of cholesterol on liposome stability and on in vitro drug release. Drug Deliv Transl Res. 2015;5(3):231-242. doi:10.1007/s13346-015-02208

29. Rog T, Pasenkiewicz-Gierula M, Vattulainen I, Karttunen M. Ordering effects of cholesterol and its analogues. Biochim Biophys Acta. 2009;1788(1):97-121. doi:10.1016/j.bbamem.2008.08.022

30. Lu RM, Chen MS, Chang DK, et al. Targeted drug delivery systems mediated by a novel Peptide in breast cancer therapy and imaging. PLoS One. 2013;8(6):e66128. doi:10.1371/journal.pone.0066128

31. Neog MK, Rasool M. Targeted delivery of p-coumaric acid encapsulated mannosylated liposomes to the synovial macrophages inhibits osteoclast formation and bone resorption in the rheumatoid arthritis animal model. Eur J Pharm Biopharm. 2018;133:162-175. doi:10.1016/j.ejpb.2018.10.010

32. Liu M, Jin S, Yan H, Du S. Effect of oxymatrine HSPC liposomes on improving bioavailability, liver target distribution and hepatoprotective activity of oxymatrine. Eur J Pharm Sci. 2017;104:212-220. doi:10.1016/j.ejps.2017.03.048

33. Hamidzadeh K, Christensen SM, Dalby E, Chandrasekaran P, Mosser DM. Macrophages and the recovery from acute and chronic inflammation. Annu Rev Physiol. 2017;79:567-592. doi:10.1146/ annurev-physiol-022516-034348

34. Ghafari M, Haghiralsadat F, Khanamani Falahati-Pour S, Zavar Reza J. Development of a novel liposomal nanoparticle formulation of cisplatin to breast cancer therapy. J Cell Biochem. 2020. doi:10.1002/jcb.29651

35. Jia $\mathrm{Y}$, Long $\mathrm{S}$, Jiang $\mathrm{N}$, et al. Oxymatrine ameliorates agomelatine-induced hepatocyte injury through promoting proteasome-mediated CHOP degradation. Biomed Pharmacother. 2019;114:108784. doi:10.1016/j.biopha.2019.108784

36. Jiang X, Xie L, Huang C, et al. Oral oxymatrine for hepatitis B cirrhosis: a systematic review protocol. Medicine (Baltimore). 2018;97(49):e13482. doi:10.1097/MD.0000000000013482

37. Zhou H, Shi HJ, Yang J, et al. Efficacy of oxymatrine for treatment and relapse suppression of severe plaque psoriasis: results from a single-blinded randomized controlled clinical trial. Br J Dermatol. 2017;176(6):1446-1455. doi:10.1111/bjd.15316

38. Zhang $\mathrm{Y}$, Yan $\mathrm{R}, \mathrm{Hu}$ Y. Oxymatrine inhibits lipopolysaccharide-induced inflammation by down-regulating Toll-like receptor 4/nuclear factor-kappa B in macrophages. Can J Physiol Pharmacol. 2015;93(4):253-260. doi:10.1139/cjpp-20140362

39. Wei K, Dai J, Wang Z, et al. Oxymatrine suppresses IL-1 $\beta$-induced degradation of the nucleus pulposus cell and extracellular matrix through the TLR4/NF- $\kappa \mathrm{B}$ signaling pathway. Exp Biol Med (Maywood). 2020;1535370219900773.

40. Zhang Z, Liu Q, Zang H, Shao Q, Sun T. Oxymatrine protects against l-arginine-induced acute pancreatitis and intestine injury involving Th1/Th17 cytokines and MAPK/NF-кB signalling. Pharm Biol. 2019;57(1):595-603. doi:10.1080/13880209.2019.1657906

\section{Publish your work in this journal}

Drug Design, Development and Therapy is an international, peerreviewed open-access journal that spans the spectrum of drug design and development through to clinical applications. Clinical outcomes, patient safety, and programs for the development and effective, safe, and sustained use of medicines are a feature of the journal, which has also been accepted for indexing on PubMed Central. The manuscript management system is completely online and includes a very quick and fair peer-review system, which is all easy to use. Visit http://www. dovepress.com/testimonials.php to read real quotes from published authors. 